\title{
Selección de una especie de Trichogramma (Hym., Trichogrammatidae) para el control biológico de la polilla de la patata Phthorimaea operculella (Lep., Gelechiidae) mediante el estudio del comportamiento de parasitación del huésped
}

\author{
J.R. Gallego, L. Mellado-López y T. Cabello* \\ Escuela Superior de Ingeniería, Departamento de Biología y Geología, Universidad de Almería, Ctra. \\ Sacramento s/n, CP. 04120, Almería, España
}

\begin{abstract}
Resumen
Las plagas que actualmente ocasionan mayores pérdidas económicas en el cultivo de patata en España son las denominadas "polilla común de la patata", Phthorimaea operculella, y "polilla guatemalteca de la patata", Tecia solanivora (Lepidoptera: Gelechiidae). Por ello, se ha llevado a cabo un estudio, en condiciones de laboratorio, para evaluar el potencial biológico de dos especies de parasitoides de huevos: Trichogramma achaeae y T. cacoeciae (Hym.: Trichogrammatidae) como agentes de control para P. operculella. Se han realizado tres grupos de ensayos: "no elección" de huésped, "elección" de huésped y estudio del comportamiento de parasitación. En todos los ensayos se compararon los huevos de la plaga frente al huésped de cría de los parasitoides: Ephestia kuehniella. En los resultados del ensayo de "no elección" se ha encontrado que $T$. achaeae parasitó significativamente más huevos huéspedes de ambas especies que $T$. cacoeciae. Así, la mortalidad total de huevos en $P$. operculella fue del $92,78 \pm 13,47 \%$ para $T$. achaeae frente al $70,88 \pm 15,11 \%$ para $T$. cacoeciae. A su vez, en el ensayo de "elección" se ha encontrado que las hembras adultas de ambas especies de parasitoides presentaron una preferencia por los huevos de $P$. operculella $\left(\beta_{2}=0,58 \pm 0,10\right.$ y $\beta_{2}=0,61 \pm 0,17$ para $T$. achaeae y $T$. cacoeciae, respectivamente). Por último, en el ensayo de comportamiento de parasitación, se ha encontrado que $T$. cacoeciae presentó significativamente menores tiempo de búsqueda y menores tiempos de manipulación de ambos huevos huéspedes que $T$. achaeae. Ambas especies de Trichogramma parecen ser buenos candidatos como agentes de control biológico de $P$. operculella, lo que debe ser confirmado posteriormente mediante estudios en campo y en almacén.
\end{abstract}

Palabras clave: Parasitoides oófagos, selección de huésped, aceptación de huésped, tiempo de búsqueda, tiempo de manipulación.

Selection of a Trichogramma species (Hym., Trichogrammatidae) for the biological control of the potato moth Phthorimaea operculella (Lep., Gelechiidae) by host-parasitism behavioral study

\begin{abstract}
Currently, the "potato tuberworm", Phthorimaea operculella, and the "Guatemalan potato moth", Tecia solanivora (Lep.: Gelechiidae) are the two pests of the greatest economic importance in Spanish potato crops. For this reason, the potential as biological control agent of two egg parasitoid species -Tri-
\end{abstract}

\footnotetext{
* Autor para correspondencia: tcabello@ual.es

Cita del artículo: Gallego JR, Mellado-López L, Cabello T (2020). Selección de una especie de Trichogramma (Hym., Trichogrammatidae) para el control biológico de la polilla de la patata Phthorimaea operculella (Lep., Gelechiidae) mediante el estudio del comportamiento de parasitación del huésped. ITEA-Información Técnica Económica Agraria 116(1): 2-18. https://doi.org/10.12706/itea.2019.013
} 
chogramma achaeae and $T$. cacoeciae (Hym.: Trichogrammatidae)- of the pest species $P$. operculella has been studied, under laboratory conditions. Three groups of trials have been carried out: "non-choice" test, "choice" test, and study of parasitic behavior. In all the trials the eggs of the pest specie were compared with the rearing host of the parasitoids: Ephestia kuehniella. In the results, "no choice" test, $T$. achaeae parasitized significantly more eggs of both species than $T$. cacoeciae. Thus, total egg mortality in $P$. operculella was $92.78 \pm 13.47 \%$ by $T$. achaeae versus $70.88 \pm 15.11 \%$ by $T$. cacoeciae. In turn, in the "choice" test, it was found that adult females of both parasitoid species preferred $P$. operculella eggs ( $\beta_{2}=0.58 \pm 0.10$ y $\beta_{2}=0.61 \pm 0.17$ para $T$. achaeae y $T$. cacoeciae, respectively). Finally, in the parasitic behavior test, it was found that $T$. cacoeciae showed significantly shorter host searching times and shorter host handling times than $T$. achaeae. Both Trichogramma species seem to be good candidates as biological control agents of $P$. operculella, which must be subsequently confirmed by field and warehouse trials.

Keywords: Egg parasitoids, host selection, host acceptance, foraging behavior, searching time, handling time.

\section{Introducción}

La patata (Solanum tuberosum L.) está considerada como uno de los cuatro cultivos más importantes para la alimentación humana, con una producción mundial de 195 millones de toneladas y una superficie cultivada de 19 millones de hectáreas (FAOSTAT, 2018).

Las plagas que actualmente ocasionan mayores pérdidas económicas en el cultivo de patata en España son las denominadas "polilla común de la patata", Phthorimaea operculella (Zeller) (Lep.: Gelechiidae), y "polilla guatemalteca de la patata", Tecia solanivora (Povolný) (Lep.: Gelechiidae).

De ellas, $T$. solanivora fue detectada en Canarias en 1999, llegando a producir pérdidas cercanas al $50 \%$ de la producción total en algunas campañas (Trujillo-García y Perera-González, 2017). En la Península, esta plaga se detectó por primera vez en septiembre de 2015 en varios términos municipales de las provincias de A Coruña y Lugo (Galicia); así como en el Principado de Asturias (MAPAMA, 2017).

A su vez, la "polilla común de la patata" es una de las plagas más importantes de los cultivos de patata a nivel mundial, pudiendo llegar a producir hasta un $80 \%$ de pérdidas en campo, así como daños importantes durante el almacenamiento (Rondon, 2010).
Es una especie cosmopolita que está ampliamente extendida en los cinco continentes (EPPO, 2015); así como en todas las zonas de cultivo de patata del territorio español.

En España, $P$. operculella se citó por primera vez en el año 1925 en Valencia, aunque posiblemente llevase más tiempo en la Península Ibérica (García-Mercet, 1925). Sus larvas forman galerías en el interior de los tubérculos, inutilizándolos para el consumo. Además, perforan los tallos y hojas de la planta, debilitándola y reduciendo los rendimientos, aunque los daños más importantes se producen después de la cosecha, cuando se almacenan los tubérculos (Kroschel y Schaub, 2013).

Hasta la fecha, el control químico con insecticidas ha dado buenos resultados para controlar la "polilla común de la patata" en campo y en almacenamiento (Trujillo-García y Perera-González, 2014). Sin embargo, la aparición de resistencias a insecticidas, conjuntamente con la cada vez más restrictiva lista de sustancias activas permitidas y la importancia de los residuos químicos en las hortalizas, hace que cada vez se tengan más en cuenta otros tipos de alternativas, como el uso de agentes de control biológico (PereraGonzález et al., 2009).

Las liberaciones de himenópteros parasitoides oófagos del género Trichogramma Westwood 
(Hym., Trichogrammatidae), constituyen un importante componente en los programas de manejo integrado de plagas para el control biológico de lepidópteros (Mills, 2010). Así, las especies de Trichogramma son los parasitoides más empleados como agentes de control biológico de especies plaga en cultivos y masas forestales a nivel mundial debido a su efectividad, a la facilidad para su producción en biofábricas a bajos costes y a su amplio rango de especies huésped (Cabello, 1985). Varias especies de este género son liberadas anualmente en el mundo en más de 33 millones de hectáreas de diferentes cultivos; ello para el control biológico de diversas especies plaga de lepidópteros (Hajek y Eilenberg, 2018).

Por otra parte, existen pocos antecedentes disponibles sobre aspectos relacionados con especies de Trichogramma como parasitoides de $P$. operculella. Se ha citado el potencial biótico de algunas especies de este género para controlar la plaga, como p. ej. T. pretiosum (Riley), T. cacoeciae (Marchal), T. evanescens (Westwood) y T. brasiliensis (Ashmead) (Harwalkar et al., 1987; Pratissoli y Parra, 2000; Saour, 2004). Además, especies no identificadas de este género han aparecido en prospecciones de enemigos naturales de la "polilla de la patata" en Colombia (Kroschel y Schaub, 2013).

No es la primera vez que se utilizan especies de Trichogramma en el control biológico de la "polilla común de la patata" en España. Así, Urquijo (1944) introdujo desde Estados Unidos de América y llevó a cabo la cría en masa de $T$. minutum y $T$. pretiosum en la antigua Estación Fitopatológica de A Coruña. Realizó sueltas de $T$. minutum en más de 44 parcelas con cultivo comercial en las zonas costeras de Galicia, desde Muros (A Coruña) hasta la desembocadura del Miño (Pontevedra). Los resultados, en los casos en los que se dispuso de datos, fueron muy buenos con un resultado favorable de control en el
$54,6 \%$ de los casos, no favorable en el $29,5 \%$ y dudoso en el resto (15,9\%). Asimismo, este autor destacó la importancia de la coincidencia de dichas liberaciones con los máximos de oviposición de los adultos de la "polilla común de la patata".

En el presente trabajo, dos especies autóctonas de Trichogramma fueron evaluadas en condiciones de laboratorio mediante el estudio del parasitismo, emergencia, desarrollo, elección de huésped en condiciones de "no elección" y "elección", así como por el estudio del comportamiento de parasitación. Todo ello con el objetivo de seleccionar la especie de parasitoide de huevos con mejor potencial biótico para el control de la "polilla común de la patata". En este sentido, hay que señalar que el parasitismo y la idoneidad de los huevos de especies plaga, usualmente determinado mediante el porcentaje de huevos parasitados y el número de adultos emergidos, entre otros parámetros, son fundamentales para la selección de un buen agente de control biológico (Hou et al., 2018).

\section{Material y métodos}

\section{Material biológico y condiciones experimentales}

Los ejemplares de los parasitoides $T$. achaeae y T. cacoeciae, utilizados en los ensayos, se obtuvieron de poblaciones naturales de huevos parasitados de lepidópteros y hemípteros Scutelleridae en las localizaciones de Fuencaliente

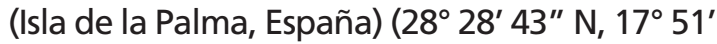
$42^{\prime \prime}$ W) y Sierra de Cazorla (Jaén, España) (37 $47^{\prime} 43,2^{\prime \prime}$ N, 2 54' 26,9" W), respectivamente. Posteriormente, dichas especies fueron mantenidas artificialmente en el laboratorio de Entomología Agrícola de la Universidad de Almería durante 9 y 6 años, respectivamente, antes del inicio de los ensayos. 
Para la cría se utilizaron huevos de la "poliIla mediterránea de la harina", Ephestia kuehniella (Zeller) (Lep., Pyralidae), siguiendo la metodología descrita por Cabello (1985). Para ello, en dos cartulinas de $2 \times 7 \mathrm{~cm}$, se adhirieron aproximadamente 2000 huevos con agua y se introdujeron en tubos de plástico de $130 \mathrm{ml}$ donde se encontraban los parasitoides adultos en proporción de 1:4 (adultos del parasitoide:huevos huéspedes), como alimento se aportó una línea fina de miel aplicada con una aguja.

La población de $P$. operculella, fue criada en el mismo laboratorio y se inició con ejemplares suministrados por el Laboratorio de Sanidad Vegetal de Almería (Junta de Andalucía, España). La cría se realizó siguiendo la metodología descrita por Fenemore (1977) utilizando patatas pequeñas, tipo guarnición, como alimento para las larvas. Para que se iniciase el desarrollo larvario sobre las patatas, discos de papel de filtro con huevos de la polilla provenientes de las cámaras de apareamiento y oviposición de los adultos (aproximadamente 20 parejas de adultos confinadas en vasos de plástico de $1000 \mathrm{ml}$ de volumen cerrados con goma elástica y gasa quirúrgica) se dejaban en contacto con los tubérculos previamente agujereados superficialmente para facilitar la penetración de las larvas neonatas. Además, disponían de una capa de vermiculita para favorecer su posterior transformación en pupa. Una vez completado el desarrollo larvario, el sustrato era tamizado para retirar las pupas y colocarlas nuevamente en cámaras de oviposición hasta la emergencia de los adultos.

En todos los casos anteriores, las poblaciones de insectos fueron criadas en condiciones climáticas controladas a $25 \pm 1{ }^{\circ} \mathrm{C}, 60-80 \%$ de humedad relativa (H.R.) y fotoperiodo de 16:8 (L:O). A su vez, los huevos de E. kuehniella (irradiados con luz UV) empleados en los diferentes ensayos fueron suministrados semanalmente por la empresa Biotop $₫(V a l-$ bonne, Francia).

\section{Ensayo de "no elección" de huésped}

Se realizó un primer ensayo del tipo "no elección" en el que se evaluaron la aceptación y parasitación por parte de las dos especies de Trichogramma sobre $P$. operculella y E. kuehniella (este último, huésped habitual de cría). Para ello, se utilizaron hembras de $T$. achaeae (previamente apareadas) o $T$. cacoeciae que fueron colocadas individualmente en tubos de ensayo $(7 \mathrm{~cm} \times 1 \mathrm{~cm}$ de diámetro). A continuación, en cada tubo de ensayo se introdujo una cartulina blanca $(0,9 \mathrm{~cm}$ $x 5,0 \mathrm{~cm}$ ) que llevaba adheridos 15 huevos de E. kuehniella o de $P$. operculella; también, en dicho momento, se aportó (sobre las paredes del tubo de ensayo) unas gotas de miel (como alimento de las hembras). Los tubos de ensayo fueron posteriormente tapados con algodón y a las hembras se les permitió parasitar durante 24 horas, en las condiciones ambientales anteriormente indicadas.

El diseño experimental fue univariante factorial, totalmente aleatorio, con dos factores implicados: especie de parasitoide (a dos niveles: $T$. achaeae y $T$. cacoeciae) y especie de huevo huésped (a dos niveles: P. operculella y $E$. kuehniella); el número de repeticiones fue variable de 10 a 19 por tratamiento. Al final del ensayo, los datos recogidos fueron: (1) porcentaje de parasitismo aparente (huevos huéspedes parasitados que viraron a color negro; respecto a los huevos no parasitados que quedaban de color blanco-anacarado), (2) porcentaje de mortalidad total (por parasitismo y por depredación; en este caso, por alimentación en el huevo huésped, "host-feeding", por parte de la hembra, sin llegar a ser parasitados) y (3) emergencia de adultos del parasitoide correspondiente $\left(F_{1}\right)$. Los valores del número de huevos huéspedes parasitados fueron analizados estadísticamente mediante modelo lineal generalizado (MLGZ) con la distribución de Poisson y la función de enlace logaritmo; a su vez, los valores medios 
fueron comparados por pares mediante el test de Wald. Para ello se usó el software estadístico IBM SPSS versión 23.

\section{Ensayo de "elección" de huésped}

El segundo ensayo fue del tipo "elección". Para ello, se siguió la misma metodología anteriormente señalada, con la excepción de que a cada hembra aislada de Trichogramma se le ofrecieron, simultáneamente, huevos de los dos huéspedes, E. kuehniella y P. operculella, a las densidades de 8 huevos de cada una de ellas. El diseño experimental fue univariante factorial, totalmente aleatorio, con dos factores implicados: especie de parasitoide (a dos niveles: $T$. achaeae y $T$. cacoeciae) y especie de huevo huésped (a dos niveles: P. operculella y $E$. kuehniella). Al final del ensayo se registró el número de huevos huéspedes parasitados. Estos datos se analizaron estadísticamente mediante el modelo lineal generalizado (MLGZ). Las medias se compararon usando el mismo test y software estadístico antes indicado. En este ensayo, el número de repeticiones por tratamiento fue de 20 .

Se evaluó también la preferencia de las hembras de cada especie de Trichogramma por los huevos de las dos especies huéspedes. Para ello se utilizó el índice de preferencia de Manly $\left(\beta_{2}\right)$ (Manly et al., 1972). La expresión de dicho índice, según Chesson (1983), fue la siguiente:

$$
\beta_{2}=\frac{\operatorname{Ln} \frac{\left(n_{i}-r_{i}\right)}{n_{i}}}{\sum_{i=1}^{i=2} \operatorname{Ln} \frac{\left(n_{i}-r_{i}\right)}{n_{i}}}
$$

donde $\beta_{2}$ es el índice de preferencia de Manly (sin reposición), $r_{i}$ es el número de huevos huéspedes parasitados y $n_{i}$ es el número de huevos huéspedes ofertados; $i=1$ y 2 , representa las dos diferentes especies de huevos huésped utilizados. Hay que señalar que cuando el valor del índice de preferencia de Manly es $\beta_{2}>0,5$ indica preferencia, indiferencia cuando $\beta_{2}=0,5$ y rechazo si $\beta_{2}<0,5$. Los valores del índice de Manly, obtenidos para cada especie de Trichogramma, especie de huésped y repetición fueron analizados estadísticamente mediante el test de Wilcoxon usando el mismo software estadístico antes indicado.

\section{Estudio del comportamiento de parasitación del huésped}

A una hembra aislada de cada especie de parasitoide se le ofreció una cantidad de 5 huevos de $E$. kuehniella o de $P$. operculella, en tandas por separado, pegados con agua en papel vegetal (círculo de diámetro $=3 \mathrm{~cm}$ ) y una distribución tipo "cinco de oros" ("patch" o parche). Para ello, cada hembra y "patch" de huevos huéspedes fueron colocados dentro de una placa Petri de vidrio (diámetro $=3 \mathrm{~cm}$ ), permitiendo la parasitación durante 1 hora.

El diseño experimental fue univariante factorial, no totalmente aleatorio, con tres factores implicados: especie de parasitoide (a dos niveles: $T$. achaeae y $T$. cacoeciae), especie de huevo huésped (a dos niveles: P. operculella y E. kuehniella) y orden de parasitación de los huevos huéspedes (a 5 niveles).

En este ensayo, los valores medidos fueron el tiempo empleado en la búsqueda del huevo huésped dentro del "patch" o parche $\left(T_{s}\right)$ y el tiempo de manipulación del huésped $\left(T_{h}\right)$, que corresponde a la suma de los tiempos empleados en la secuencia de oviposición estándar de las hembras adultas de Trichogramma: (1) primer contacto con el huésped mediante las antenas; (2) examen de la superficie del huevo huésped mediante las antenas antes de su perforación con el ovipositor; (3) perforación (punción con éxito del corion del huésped); (4) inserción completa del ovipositor y movimiento abdominal (pero 
antes del bombeo abdominal del huevo propio) y (5) oviposición (deposición del huevo propio en el huevo huésped) (Nurindah et al., 1999a).

Hay que señalar que la observación directa del comportamiento por parte de un observador humano puede causar interferencias en el comportamiento del animal observado y comprometer así la validez de los datos como ha sido señalado por Wade et al. (2005). Por este motivo y para facilitar el procesado de recogida de la información, en dichas condiciones y sin interferencia humana, se tomó una foto cada 10 segundos durante la realización de los ensayos mediante una cámara digital Eos 550D ${ }^{\circledR}$ (Canon), lente: EFS 18-55 con función macro y dotada de un tubo de extensión de $12 \mathrm{~mm}$ para permitir una menor distancia de enfoque. La cámara se conectó mediante cable a un ordenador mediante el software "communication software for the camera EOS utility", versión 2.14. Se utilizó iluminación diascópica (luz transmitida) para obtener las fotografías. Debido al tipo de lente utilizada y la resolución en píxeles necesarios, solo se pudieron capturar las imágenes de 6 arenas al mismo tiempo. Por este motivo el proceso se debió repetir durante varios días; en cada sesión de fotos se dispusieron al azar los distintos tratamientos y repeticiones utilizadas. Ya que el ensayo no pudo realizarse al mismo tiempo, las repeticiones son en realidad pseudo-repeticiones y como tales fueron tratadas en los análisis estadísticos llevados a cabo tal y como se explica más adelante. Las fotografías, después de tomadas, fueron montadas secuencialmente mediante el software Virtual Dub versión 1.49 lo que permitió la visualización del comportamiento y la anotación de los tiempos empleados por cada uno de los parasitoides en función de la especie huésped en cada repetición.

Como se ha comentado en el párrafo anterior, debido a tener que llevar a cabo el ensayo en diferentes días, las repeticiones de los tratamientos no representan verdaderas repeticiones en un diseño totalmente aleatorio. Esto es frecuente en estudios de comportamiento de insectos. Ante dicha situación no son de aplicación los clásicos análisis estadísticos mediante el análisis de la varianza (ANOVA), ni los más recientes de modelo lineal general (MLG). En cambio, sí le son de aplicación los modelos lineales generalizados (MLGZ) (Ricard, 2008). Por este motivo, se aplicaron MLGZ en los análisis estadísticos de los tiempos registrados $\left(T_{s}\right.$ y $\left.T_{h}\right)$, utilizando la función de distribución gamma y función de enlace logaritmo, con el mismo software estadístico anteriormente indicado. El número de pseudo-repeticiones fue variable de 11 a 24 por tratamiento.

\section{Resultados}

\section{Ensayo de "no elección" de huésped}

En la de la Tabla 1 (parte superior) se recogen los valores medios del número de huevos parasitados, en el ensayo de "no elección" de huésped por las hembras adultas de $T$. achaeae frente a $T$. cacoeciae, según la especie parasitada. A su vez, en la Figura 1 se representa dichos valores expresados en porcentaje.

En el análisis estadístico se presentó una alta significación estadística del modelo (Prueba Ómnibus: $\chi^{2}$ de razón de verosimilitud $=$ $10,681 ;$ g.l. $=3 ; P=0,014)$. En las pruebas de efectos del modelo se encontró significación estadística de la especie de parasitoide $\left(\chi^{2}\right.$ de razón de verosimilitud $=4,661$; g.I. $=1 ; P=$ 0,031 ) y de la especie huésped ( $\chi^{2}$ de razón de verosimilitud $=3,953$; g.I. $=1 ; P=0,047)$; pero no de su interacción ( $\chi^{2}$ de razón de verosimilitud $=1,468 ;$ g.I. $=1 ; P=0,226)$.

En la Figura 2 se muestra la mortalidad total causada por cada especie de parasitoide que 
Tabla 1. Número medio ( $\pm E E$ ) de huevos parasitados (parasitismo aparente) en los ensayos de "elección" y "no elección", cuando a las hembras de dos especies de parasitoides (T. achaeae y T. cacoeciae) se les ofertaron huevos de dos especies huéspedes ( $E$. kuehniella y $P$. operculella), llevados a cabo en condiciones de laboratorio $\left(25 \pm 2{ }^{\circ} \mathrm{C} ; 60-80 \%\right.$ de H.R. y luz continua) (* Valores, para cada ensayo, seguidos por distinta letra presenta diferencias significativas mediante test de Wald de comparación por pares: $\chi^{2}$ de Wald = 9,546; g.l. = 3; $\mathrm{P}=0,023$ y $\chi^{2}$ de Wald $=12,318 ;$ g.l. $=3 ; \mathrm{P}=0,006$, respectivamente para el primer y segundo ensayo).

Table 1. Mean number ( \pm SE) of parasitized eggs (apparent parasitism) in the "choice" and "no choice" trials, when eggs of two parasitoid species (T. achaeae and T. cacoeciae) were offered eggs of two host species (E. kuehniella and P. operculella), carried out under laboratory conditions $\left(25 \pm 2{ }^{\circ} \mathrm{C}, 60-80 \%\right.$ RH and continuous light) (*Values, for each test, followed by different letter shows significant differences by Wald $\chi^{2}$ pairwise test: $\chi^{2}$ de Wald $=9.546$, g.l. $=3, P=0.023$ y $\chi^{2}$ de Wald $=12.318$, g.l. $=3, P$ $=0.006$, respectively for the first and second trial).

\begin{tabular}{|c|c|c|c|c|c|}
\hline \multirow{3}{*}{ Ensayo } & \multirow{3}{*}{ Parasitoide } & \multicolumn{4}{|c|}{ Parasitismo aparente (Media \pm EE) } \\
\hline & & \multicolumn{2}{|c|}{ E. kuehniella } & \multicolumn{2}{|c|}{ P. operculella } \\
\hline & & $\mathrm{N}$ & Valores & $\mathrm{N}$ & Valores \\
\hline \multirow[t]{2}{*}{ No elección } & T. achaeae & 10 & $9,10 \pm 0,95 b$ & 12 & $12,00 \pm 1,00 a$ \\
\hline & T. cacoeciae & 19 & $8,37 \pm 0,66 b$ & 19 & $8,95 \pm 0,69 b$ \\
\hline \multirow[t]{2}{*}{ Elección } & T. achaeae & 20 & $4,20 \pm 0,46 c$ & 20 & $5,10 \pm 0,51 b c$ \\
\hline & T. cacoeciae & 20 & $5,70 \pm 0,53 a b$ & 20 & $6,70 \pm 0,58 a$ \\
\hline
\end{tabular}

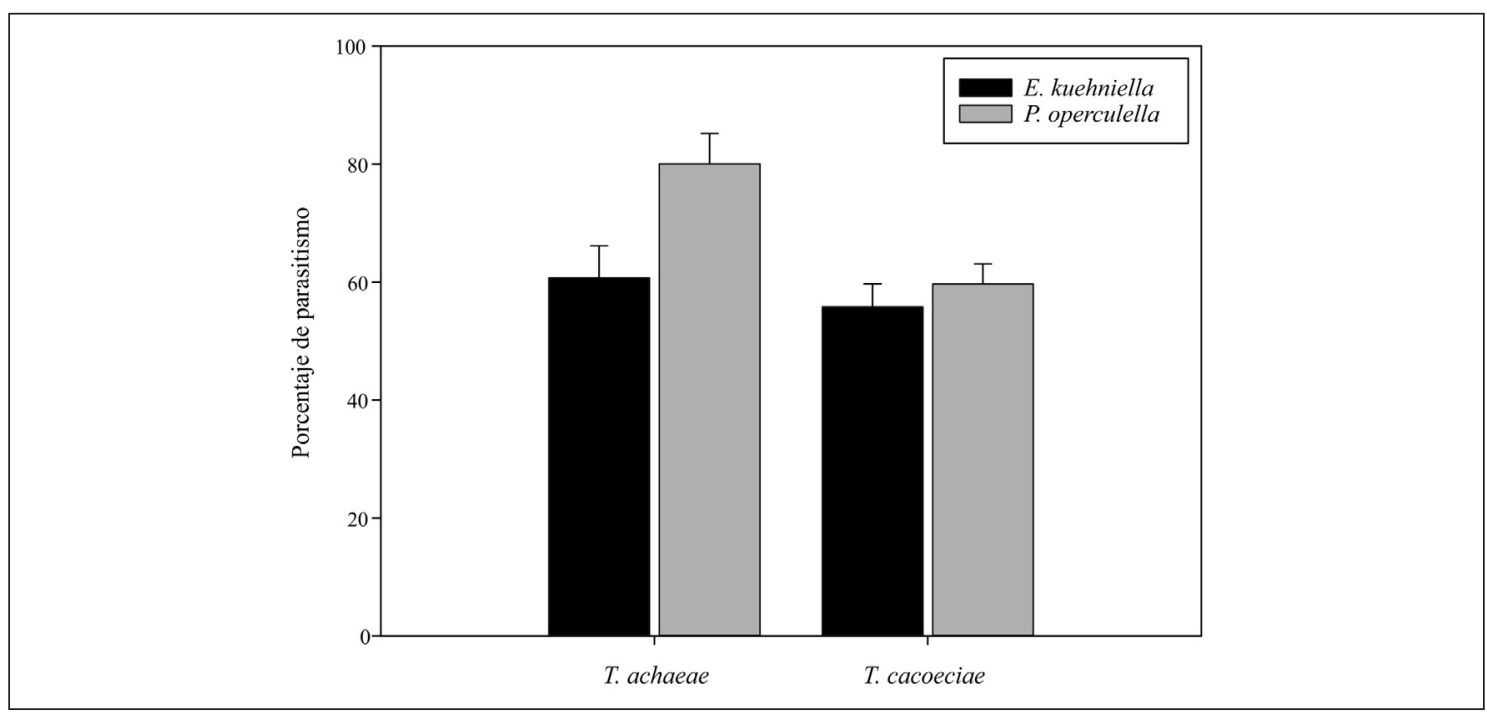

Figura 1. Porcentajes medios de parasitismo de los huevos huéspedes de $E$. kuehniella y P. operculella, causado por las hembras adultas de $T$. achaeae y $T$. cacoeciae en el ensayo de "no elección" de huésped, llevado a cabo bajo condiciones de laboratorio $\left(25 \pm 2{ }^{\circ} \mathrm{C}, 60-80 \%\right.$ H.R. y luz continua) (Líneas verticales indican los errores estándares de las medias).

Figure 1. Average percentages of parasitism of the host eggs: E. kuehniella and P. operculella by the adult females of T. achaeae and T. cacoeciae in the host "no choice" test, carried out under laboratory conditions (25 $\pm 2{ }^{\circ} \mathrm{C}, 60-80 \% \mathrm{RH}$ and continuous light) (Vertical lines indicate the standard errors of the means). 


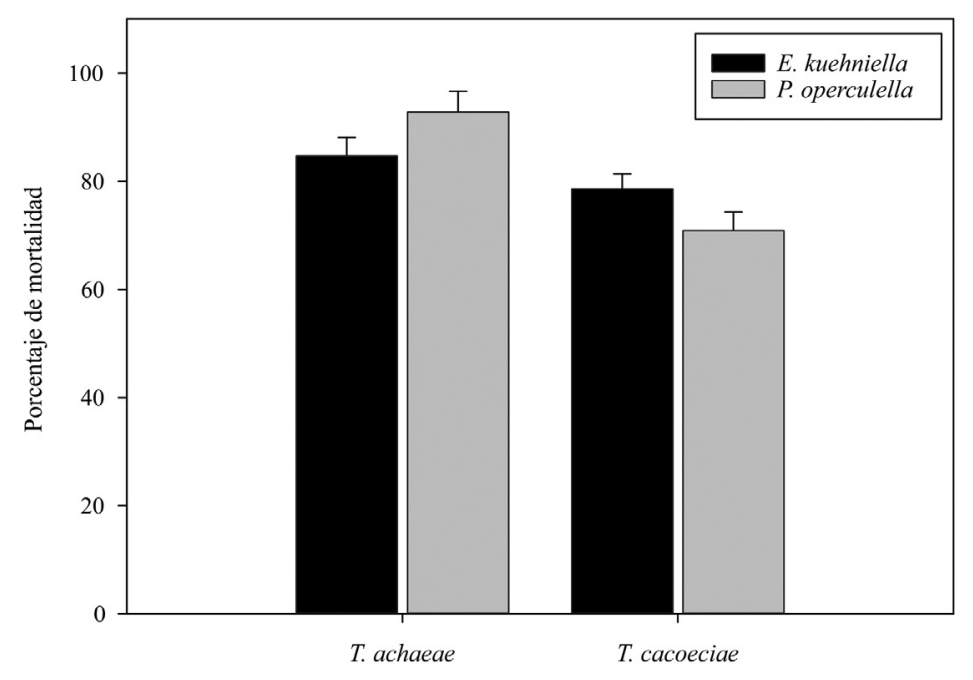

Figura 2. Porcentajes medios de mortalidad total (parasitismo + depredación por alimentación) en huevos huéspedes de $E$. kuehniella y P. operculella, causado por las hembras de $T$. achaeae y $T$. cacoeciae en el ensayo de "no elección" de huésped, llevado a cabo en condiciones de laboratorio $\left(25 \pm 2{ }^{\circ} \mathrm{C} ; 60-80 \%\right.$ H.R. y luz continua) (Líneas verticales indican los errores estándares de las medias).

Figure 2. Average percentages of total mortality (parasitism + host feeding) in host eggs of E. kuehniella and P. operculella, by females of T. achaeae and T. cacoeciae in the host "no choice" test, carried out in laboratory conditions $\left(25 \pm 2{ }^{\circ} \mathrm{C}, 60-80 \% \mathrm{RH}\right.$ and continuous light) (Vertical lines indicate the standard errors of the means).

engloba a los huevos muertos a consecuencia del parasitismo y de la depredación o "host-feeding", en ambos casos por hembra. Dicha mortalidad total fue mayor para $T$. achaeae, independientemente del tipo de huésped. Para los huevos huéspedes de la "polilla común de la patata" dicho valor fue del $92,78 \pm 13,47 \%$ para $T$. achaeae frente al $70,88 \pm 15,11 \%$ para $T$. cacoeciae.

En la Figura 3 se muestra la emergencia de adultos según especie de huésped y especie de Trichogramma empleada. El porcentaje de adultos emergidos (generación $F_{1}$ ) fue mayor para la especie $T$. achaeae que para $T$. cacoeciae.

Se puede observar que las hembras adultas de ambas especies de parasitoides acepta- ron como huésped y consiguieron un desarrollo completo en los huevos de la plaga $P$. operculella (Tabla 1, Figura 3).

\section{Ensayo de "elección" de huésped}

Los datos obtenidos para el número de huevos parasitados en el ensayo de "elección" (preferencia) se recogen en la Tabla 1 (parte inferior). En el análisis estadístico se presentó una alta significación estadística del modelo (Prueba Ómnibus: $\chi^{2}$ de razón de verosimilitud $=12,247$; g.l. $=3, P=0,007$ ). En las pruebas de efectos del modelo se encontró también alta significación estadística de la especie de parasitoide ( $\chi^{2}$ de razón de verosimilitud $=8,906 ;$ g.I. $=1 ; P=0,003)$; pero no de la especie huésped ( $\chi^{2}$ de razón de vero- 


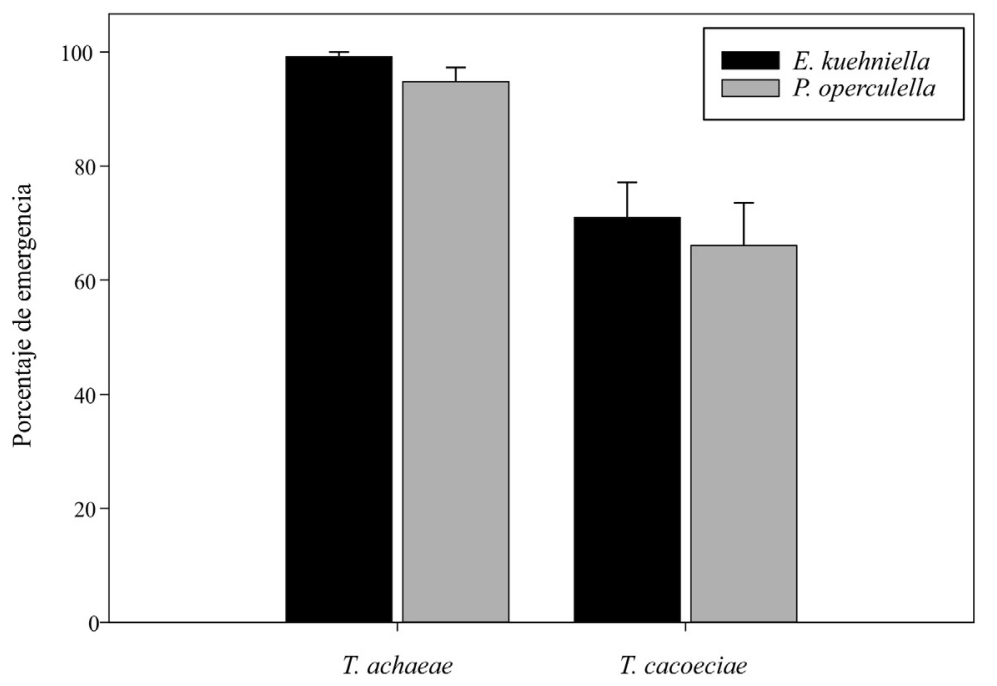

Figura 3. Porcentajes medios de emergencia de $T$. achaeae y $T$. cacoeciae en función del huésped (E. kuehniella y $P$. operculella) parasitado previamente por las hembras adultas en el ensayo de "no elección", llevado a cabo en condiciones de laboratorio $\left(25 \pm 2{ }^{\circ} \mathrm{C} ; 60-80 \%\right.$ H.R. y luz continua) (Líneas verticales indican los errores estándares de las medias).

Figure 3. Average emergency of T. achaeae and T. cacoeciae adults, according to the host egg (E. kuehniella and P. operculella) previously parasitized by the adult females in the "no choice" test, carried out under laboratory conditions $\left(25 \pm 2^{\circ} \mathrm{C}\right) 60-80 \%$ RH and continuous light) (Vertical lines indicate the standard errors of the means).

similitud $=3,350 ;$ g.I. $=1 ; P=0,067)$ ni de su interacción $\left(\chi^{2}\right.$ de razón de verosimilitud $=$ 0,$028 ;$ g.I. $=1 ; P=0,867$ ).

Puede observarse cómo $T$. cacoeciae parasitó significativamente un mayor número de huevos de $P$. operculella $(6,70 \pm 0,58)$ respecto a $T$. achaeae $(5,10 \pm 0,51)$.

Las hembras adultas de $T$. achaeae presentaron una preferencia por los huevos de $P$. operculella $\left(\beta_{2}=0,58 \pm 0,10\right)$ respecto a los de E. kuehniella $\left(\beta_{2}=0,42 \pm 0,10\right)(Z=-2,772 ; P$ $=0,006)$. Similar preferencia mostraron las hembras adultas de $T$. cacoeciae por los huevos de $P$. operculella $\left(\beta_{2}=0,61 \pm 0,17\right)$ respecto a los de $E$. kuehniella $\left(\beta_{2}=0,39 \pm 0,17\right)$ $(Z=-2,501 ; P=0,012)$.

\section{Comportamiento de parasitación}

Los valores de $T_{s}$ y $T_{h}$ encontrados para las dos especies de Trichogramma en relación al huésped $E$. kueniella se recogen en las Figuras $4 \mathrm{~A}$ y $5 \mathrm{~A}$. A su vez, los valores correspondientes para $P$. operculella se muestran en las Figuras $4 \mathrm{~B}$ y $5 \mathrm{~B}$.

En el análisis estadístico mediante MLGZ del tiempo de búsqueda $\left(T_{s}\right)$ se encontró una alta significación del modelo (Prueba ómnibus: $\chi^{2}$ de razón de verosimilitud $=324,882$; g.l. $=19 ; P<0,0001)$; en las pruebas de los efectos del modelo se encontraron efectos altamente significativos de la especie de parasitoide $\left(\chi^{2}\right.$ de razón de verosimilitud $=$ $124,754 ;$ g.I. $=1 ; P<0,0001)$, especie huésped $\left(\chi^{2}\right.$ de razón de verosimilitud $=139,421 ;$ g.l. 

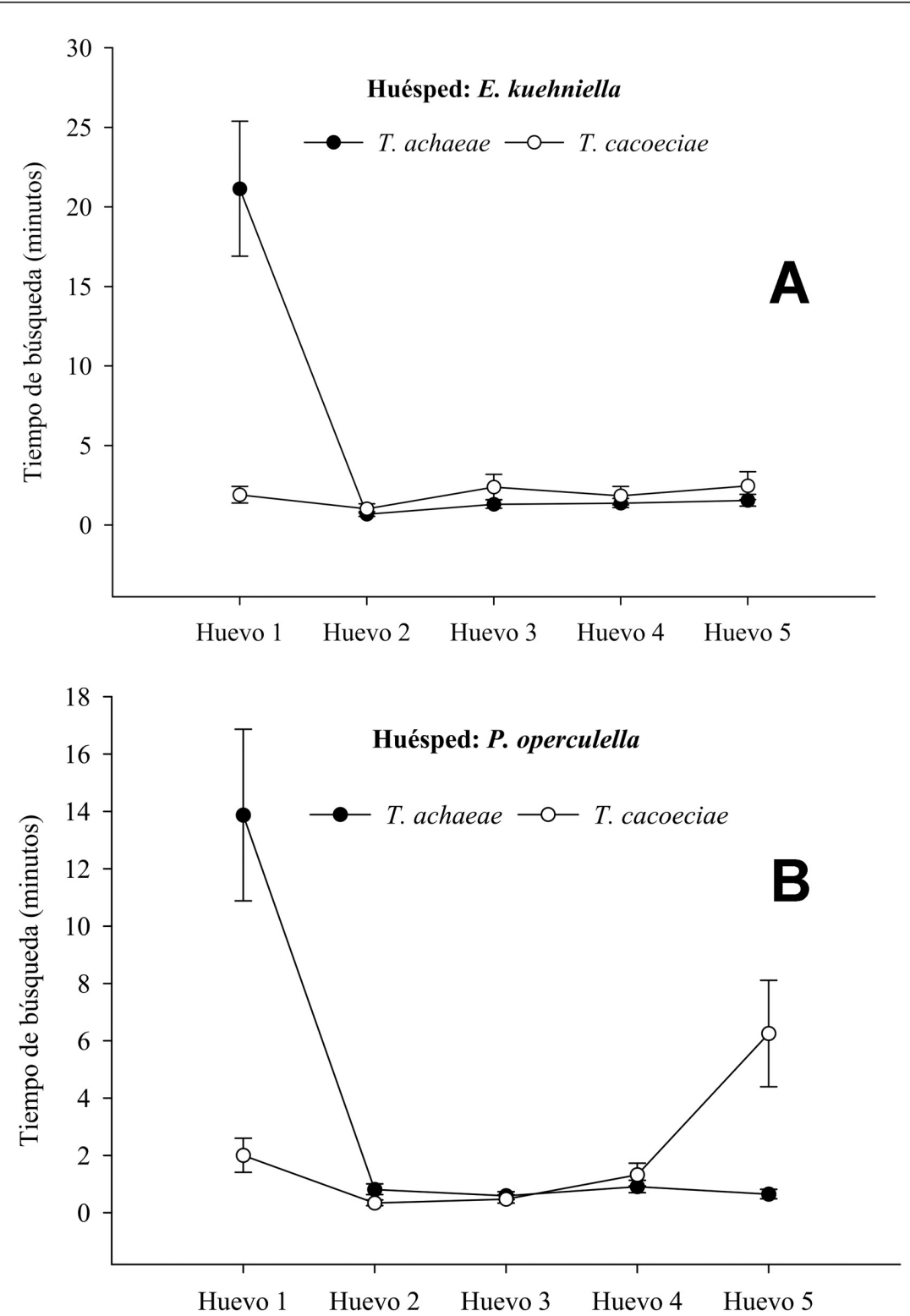

Figura 4. Variación del tiempo medio de búsqueda del huésped $\left(T_{s}\right)$, expresados en minutos, de las hembras adultas de $T$. achaeae y $T$. cacoeciae, según secuencia de oviposición, en el caso de la especie huésped E. kuehniella (A) y P. operculella (B) en condiciones de laboratorio $\left(25 \pm 1^{\circ} \mathrm{C}, 60-80 \%\right.$ H.R. y luz continua) (líneas verticales indican los errores estándares de las medias).

Figure 4. Progression of the average of searching time $\left(T_{s}\right)$ per egg host, in minutes, of the adult females of T. achaeae and T. cacoeciae, according to the oviposition sequence, in the case of the host species E. kuehniella $(A)$ and P. operculella $(B)$ under laboratory conditions $\left(25 \pm 1{ }^{\circ} \mathrm{C}, 60-80 \% R H\right.$ and continuous light) (vertical lines indicate the standard errors of the means). 
$=1 ; P<0,0001)$ y orden de parasitación del huésped ( $\chi^{2}$ de razón de verosimilitud $=$ 341,602; g.I. $=4 ; P<0,0001)$, así como de las interacciones parasitoide* huésped $\left(\chi^{2}\right.$ de razón de verosimilitud $=124,754 ;$ g.l. $=1 ; P<$ $0,0001)$, parasitoide*orden $\left(\chi^{2}\right.$ de razón de verosimilitud $=237,311$; g.I. $=4 ; P<0,0001)$, huésped*orden $\left(\chi^{2}\right.$ de razón de verosimilitud $=135,774 ;$ g.l. $=4 ; P<0,0001)$ y parasitoide*huésped*orden $\left(\chi^{2}\right.$ de razón de verosimilitud $=148,462 ;$ g.I. $=4 ; P<0,0001)$. De sus análisis se observa un comportamiento de búsqueda del huésped muy diferente entre ambas especies de parasitoides, así como el hecho de estar muy condicionado por la especie de huevo parasitado y el orden de parasitación. No obstante, a efectos globales, los valores medios del tiempo de búsqueda no presentaron diferencias significativas entre las dos especies de parasitoides $\left(\chi^{2}\right.$ de Wald $=0,763 ;$ g.l. $=1 ; P=0,382$ ). Así, el valor medio para $T$. achaeae fue de 1,67 $\pm 0,12$ min y para $T$. cacoeciae de 1,51 $\pm 0,15 \mathrm{~min}$.

Sin embargo, si se tiene en cuenta el orden de parasitación, sí se presentaron marcadas diferencias. En este sentido, hay que señalar que el $T_{s}$ del huevo parasitado en primer lugar por $T$. achaeae fue, tanto para el huevo huésped de $E$. kuehniella $(21,15 \pm 4,24 \mathrm{~min})$ como para $P$. operculella $(13,88 \pm 2,99 \mathrm{~min})$, significativamente mayor que el empleado por $T$. cacoeciae para ambos huéspedes y número de orden $(1,91 \pm 0,52$ y 2,01 $\pm 0,60 \mathrm{~min}$, respectivamente) ( $\chi^{2}$ de Wald $=111,883 ;$ g.l. $=19 ; P<0,0001)$ (Figuras 4A y B).

A su vez, en el análisis estadístico mediante MLGZ del tiempo de manipulación $\left(T_{h}\right)$ se encontró una alta significación del modelo (Prueba ómnibus: $\chi^{2}$ de razón de verosimilitud =138,780; g.l. = 19; $P<0,0001)$; en las pruebas de los efectos del modelo se encontraron efectos altamente significativos de la especie de parasitoide ( $\chi^{2}$ de razón de verosimilitud $=14,863 ;$ g.l. $=1 ; P<0,0001)$ y orden de parasitación del huésped $\left(\chi^{2}\right.$ de razón de verosimilitud $=94,188 ;$ g.I. $=4 ; P<0,0001$ ), así como de las interacciones parasitoide*huésped $\left(\chi^{2}\right.$ de razón de verosimilitud $=8,163 ;$ g.l. $=1 ; P=0,004)$, y parasitoide* orden $\left(\chi^{2}\right.$ de razón de verosimilitud $=10,073 ;$ g.l. $=4 ; P=$ $0,039)$. Sin embargo, no se encontraron efectos significativos de la especie huésped $\left(\chi^{2}\right.$ de razón de verosimilitud $=0,001 ;$ g.I. $=1 ; P=$ $0,977)$, huésped*orden ( $\chi^{2}$ de razón de verosimilitud $=3,398 ;$ g.I. $=4 ; P=0,494)$ y parasitoide*huésped*orden ( $\chi^{2}$ de razón de verosimilitud $=2,063 ;$ g.l. $=4 ; P=0,724) . A$ diferencia de lo encontrado en el $T_{s^{\prime}}$ parece que los tiempos de manipulación del huevo huésped por ambas especies de Trichogramma, presentaron menos variabilidad.

El promedio del tiempo de manipulación en función de la especie de Trichogramma fue de 4,18 $\pm 0,14 \mathrm{~min}$ para $T$. achaeae y de 3,32 $\pm 0,16$ min para $T$. cacoeciae, con diferencias significativas entre dichos valores $\left(\chi^{2}\right.$ de Wald $=16,427$; g.l. $=1 ; P<0,0001)$. Sin embargo, no hubo diferencias significativas entre los valores medios encontrados para el huevo huésped $E$. kuehniella $(3,72 \pm 0,15 \mathrm{~min})$ y el de $P$. operculella $(3,73 \pm 0,15 \mathrm{~min})\left(\chi^{2} \mathrm{de}\right.$ Wald $=0,001 ;$ g.I. $=1 ; P=0,977$ ). Ello parece indicar un comportamiento de parasitación distinto entre $T$. achaeae y $T$. cacoeciae, más debido a las características propias de las hembras de cada especie e independiente del huevo huésped. Según los datos obtenidos (Figuras $5 \mathrm{~A}$ y $B$ ), se debe señalar que las hembras de $T$. achaeae emplearon un $T_{h}(4,54$ $\pm 0,23 \mathrm{~min}$ ) en huevos de $P$. operculella significativamente mayor que las de $T$. cacoeciae (3,06 $\pm 0,20 \mathrm{~min})$; pero no con relación al otro huésped ( $\chi^{2}$ de Wald $=24,568$; g.l. $=3$; $P<0,0001)$. A su vez, se puede observar, en ambas especies de parasitoides y ambas especies de huevos huéspedes, que el valor medio del $T_{h}$ decreció a medida que las hembras adultas fueron parasitando los huevos. Así, el valor medio para todas las especies fue de 6,39 $\pm 0,39$ min para el primer huevo, dis- 

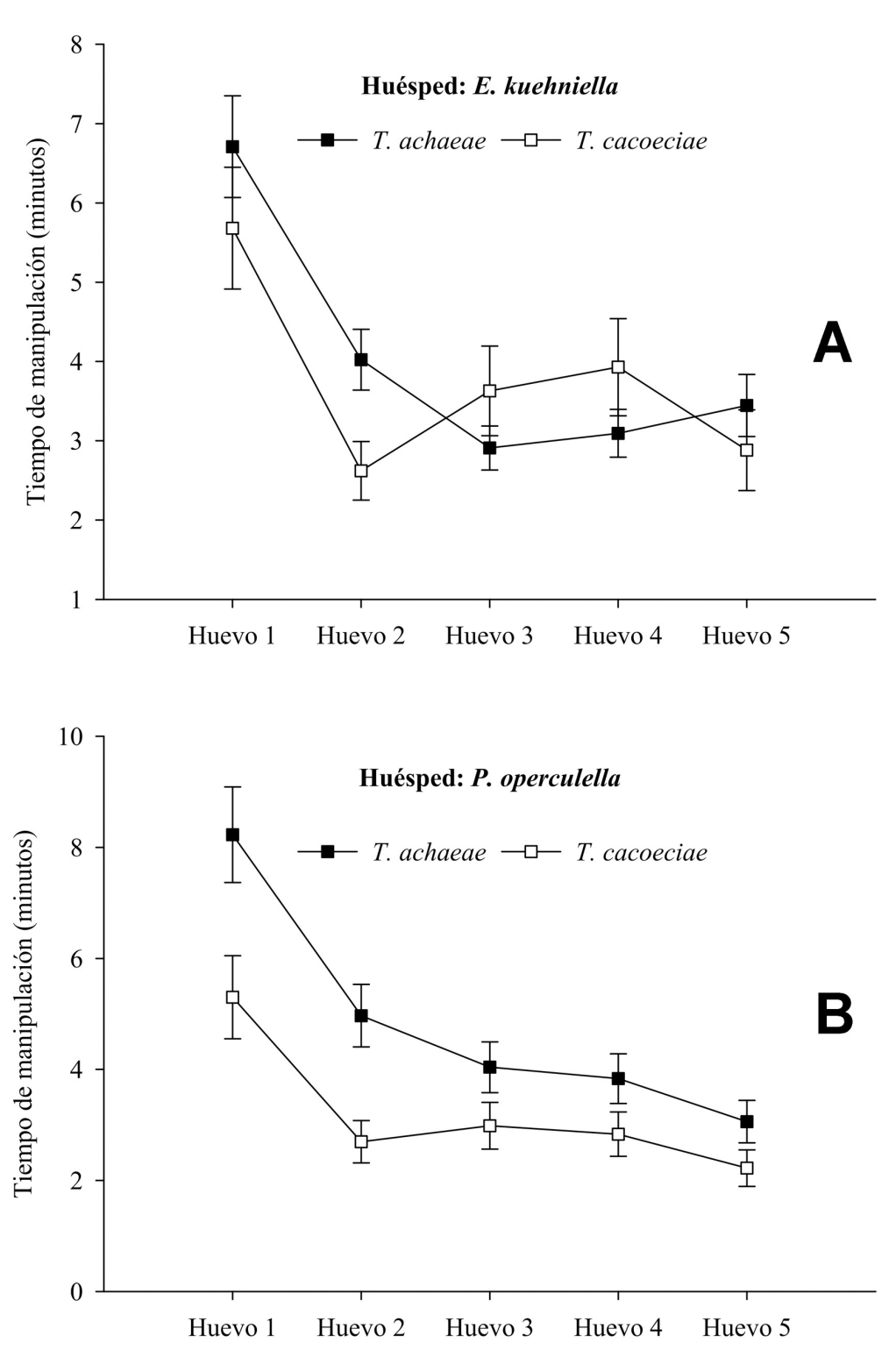

Figura 5. Variación del tiempo medio de manipulación del huésped $\left(T_{h}\right)$, expresados en minutos, en las hembras adultas de $T$. achaeae y $T$. cacoeciae, según secuencia de oviposición, en el caso de la especie huésped E. kuehniella (A) y P. operculella (B) en condiciones de laboratorio ( $25 \pm 1{ }^{\circ} \mathrm{C}, 60-80 \%$ H.R. y luz continua) (líneas verticales indican los errores estándares de las medias).

Figure 5. Progression of the average of handling time $\left(T_{h}\right)$ per egg host, in minutes, of the adult females of T. achaeae and T. cacoeciae, according to the oviposition sequence, in the case of the host species E. kuehniella and P. operculella (B) under laboratory conditions $\left(25 \pm 1^{\circ} \mathrm{C}, 60-80 \% \mathrm{RH}\right.$ and continuous light) (vertical lines indicate the standard errors of the means). 
minuyendo significativamente hasta los 2,87 $\pm 0,21$ min en el quinto huevo huésped $\left(\chi^{2}\right.$ de Wald $=66,421$; g.I. $=4 ; P<0,0001)$. Este hecho fue más acusado sobre $P$. operculella que sobre E. kuehniella (Figuras 5A y B).

\section{Discusión}

No es la primera vez que se aborda el control biológico de la "polilla común de la patata" mediante especies de Trichogramma. Así, el presente trabajo se puede considerar una continuación de dicha línea con la diferencia, en el presente caso, de que se aborda el control biológico de la especie plaga con especies autóctonas de este género de parasitoides oófagos.

Los resultados encontrados en el ensayo de "no elección" demuestran la capacidad de reconocimiento y aceptación de los huevos de $P$. operculella por parte de las especies de Trichogramma estudiadas. Para ambas especies de parasitoides, el número de huevos parasitados de la plaga supera a los del huésped habitualmente empleado para la cría, $E$. kuehniella (Tabla 1). Además, en este mismo ensayo se ha constatado cómo las dos especies de Trichogramma consiguen completar su desarrollo dentro del huevo plaga con un buen porcentaje de emergencia, lo cual es un indicativo de la idoneidad del huésped, ya que ésta hace referencia a la capacidad del parasitoide para completar el desarrollo usando el huésped en cuestión (Pak, 1990).

Por otra parte, la emergencia de adultos, en ambos huéspedes, presentó diferencias significativas en ambas especies de parasitoides (Figura 3). Con relación a este parámetro, autores como Van Lenteren (1980) no consideran la idoneidad del huésped como un criterio importante para la selección de enemigos naturales que van a ser liberados en sueltas inundativas, siempre y cuando la acción de los parasitoides conlleve la muerte del huésped, aunque éstos no lleguen a desarrollarse. Por el contrario, hay otros autores que sostienen que si los huevos plaga no mueren cuando son atacados, la emergencia debería ser considerada un criterio en la selección del enemigo natural (Pak et al., 1990; Hou et al., 2018).

En línea con estas argumentaciones, cabe destacar que la mortalidad total causada por el parasitismo y el "host feeding" es particularmente importante para $T$. achaeae, frente a $T$. cacoeciae. En ambos casos, el porcentaje de "host feeding" es similar, en torno al $12 \%$, por lo que las diferencias en la mortalidad se deben a un mayor parasitismo por parte de $T$. achaeae. En algunas especies de Trichogramma, el "host feeding" contribuye significativamente al control de la plaga (Vasquez et al., 1997). En el caso de $T$. achaeae, usado para el control de $T$. absoluta en invernaderos, se ha demostrado la importancia de la mortalidad causada por el parasitoide sin que este llegue a desarrollarse en el huevo huésped ("host-feeding"), siendo un mecanismo importante para en el control de la plaga (Cabello et al., 2009; Cabello et al., 2012).

En principio, la "polilla común de la patata" puede ser considerada como un buen huésped para Trichogramma spp., en parte debido al tamaño de su huevo; tal y como se ha indicado en los resultados, es ligeramente superior al del huésped de cría E. kuehniella que presenta valores de longitud y anchura inferiores a las 600 y $300 \mu \mathrm{m}$, respectivamente (Cabello et al., 2009).

Los resultados encontrados no nos deben extrañar ya que Trichogramma es un género compuesto por parasitoides generalistas y altamente polífagos (Cabello, 1985; Polaszek, 2010). Sin embargo, el nivel de parasitismo puede variar entre diferentes huéspedes debido a factores como la preferencia del parasitoide o el estado, tamaño y edad del huevo huésped, entre otros (Pak, 1988; Pizzol et al., 2012; Du et al., 2018). 
En el presente estudio, las dos especies de Trichogramma evaluadas prefirieron los huevos de $P$. operculella frente a los de E. kuehniella, como se refleja observando los valores de huevos parasitados con síntomas de parasitismo aparente (viran a color negro) y del índice de preferencia de Manly.

Por otra parte, parecería que los huevos de la plaga son un huésped menos idóneo que los de $E$. kuehniella debido a que emergieron un menor número de adultos de los huevos parasitados de la "polilla de la patata" frente a los del huésped de cría. Esto puede deberse a una baja calidad nutricional del huevo huésped que repercute en el desarrollo larvario de Trichogramma (Huang et al., 2018) o a la existencia de superparasitismo que puede incrementar significativamente la mortalidad de la descendencia en parasitoides oófagos, por competencia intraespecífica (Duval, 2018).

$T$. achaeae y $T$. cacoeciae aceptan bien los huevos de $P$. operculella. Es la primera vez que se cita a $T$. achaeae como potencial agente de control de esta especie plaga, según la bibliografía consultada. Por el contrario, T. cacoeciae ya ha sido evaluada en condiciones de laboratorio y con buenos resultados como potencial agente de control biológico de la "polilla común de la patata" por Saour (2004).

En los resultados obtenidos en los ensayos se ha encontrado que los tiempos de búsqueda $y$, en menor medida, los tiempos de manipulación, de las hembras adultas de $T$. cacoeciae eran menores que los correspondientes a $T$. achaeae. Dichas diferencias pueden ser debidas a la diferente forma de reproducción de ambas especies partenogénesis telitóquica y partenogénesis arrenotóquica, respectivamente. Las hembras adultas de Trichogramma, con reproducción arrenotóquica, tienen que decidir, en función del huevo huésped, tamaño del "patch", etc. (Wajnberg, 2010) si ovipositar un óvulo que no sea fecundado (que generará a un macho) o un óvulo fe- cundado (que generará a una hembra) (Wajnberg, 1993). Contrariamente, las hembras adultas de $T$. cacoeciae no tiene que hacer dicha asignación del sexo ya que siempre ovipositan óvulos con embriones diploides viables que dan lugar a hembras. Por ello, sus valores de $T_{s}$ y $T_{h}$ serían menores, como se ha encontrado en el presente trabajo.

\section{Conclusiones}

Los resultados de aceptación, desarrollo en el huésped, preferencia y comportamiento de parasitación por parte de $T$. achaeae y $T$. cacoecia sobre la "polilla común de la patata" $P$. operculella muestran que ambas especies de Trichogramma son buenas candidatas para el control biológico de la plaga con ciertas peculiaridades. Por una parte, T. achaeae parasita más, se desarrolla mejor y causa una mayor mortalidad en los huevos de la "polilla de la patata". Por otra parte, T. cacoeciae encuentra los huevos de la plaga más rápido y los parasita en menor tiempo. En vista a la complementariedad de ambas especies de Trichogramma, podría ser interesante plantear el empleo simultáneo de ellas. Ese uso simultáneo de varias especies de Trichogramma ha sido reportado con anterioridad como exitoso para el control de esta plaga en invernaderos (Zimmerman y Wührer, 2010). Sin embargo, estos resultados deben ser corroborados con posteriores estudios en condiciones de semi-campo y campo; así como en condiciones de almacenamiento de la patata.

\section{Agradecimientos}

Este trabajo ha sido financiado dentro del proyecto titulado: Desarrollo de nuevos métodos para el manejo integrado para las polillas de la patata Phthorimaea operculella y 
Tecia solanivora (Ref.: RTA2015-00074-C0200), financiado en la convocatoria de 2015 de proyectos de investigación fundamental orientada y acciones complementarias en el marco del programa estatal de I+D+l orientada a los retos de la sociedad (reto de seguridad y calidad alimentaria, actividad agraria productiva y sostenible, sostenibilidad de los recursos naturales e investigación marina y marítima). Instituto Nacional de Investigación y Tecnología Agraria y Alimentaria (INIA) y Ministerio de Economía y Competitividad. España.

\section{Conflicto de intereses}

Los autores declaran no tener ningún conflicto de intereses.

\section{Referencias bibliográficas}

Cabello T (1985). Biología de dos especies de Trichogramma (Hym.: Trichogrammatidae) parasitas de Heliothis spp. Lep.: Noctuidae) en algodonero. Posibilidades de su empleo como agente de control. Tesis Doctoral, Universidad de Córdoba, España.

Cabello T, Gallego JR, Vila E, Soler A, del Pino M, Carnero A, Hernández-Suárez $E$, Polaszek A (2009). Biological control of the South American tomato pinworm, Tuta absoluta (Lep.: Gelechiidae), with releases of Trichogramma achaeae (Hym.: Trichogrammatidae) in tomato greenhouses of Spain. IOBC/WPRS Bulletin 49: 225-230.

Cabello T, Gallego JR, Fernandez FJ, Gamez M, Vila E, del Pino M, Hernandez-Suarez E (2012). Biological control strategies for the South American tomato moth (Lep.: Gelechiidae) in greenhouse tomatoes. Journal of Economic Entomology 105: 2085-2096. https://doi.org/10.1603/ EC12221
Chesson J (1983). The estimation and analysis of preference and its relationship to foraging models. Ecology 64(5): 1297-1304. https://doi.org/10.2307/ 1937838

Du WM, Xu J, Hou YY, Lin Y, Zang LS, Yang $X$, Zhang JJ, Desneux N (2018). Trichogramma parasitoids can distinguish between fertilized and unfertilized host eggs. Journal of Pest Science 91(2): 771-780. https://doi.org/10.1007/s10340017-0919-z

Duval JF (2018). Quantification des effets du superparasitisme sur la valeur adaptative et la survie des couvées chez Trichogramma euproctidis Girault. Tesis Doctoral, Université de Montréal, Canada.

EPPO (2015). Phthorimaea operculella (PHTOOP). EPPO Global Database. Disponible en: https:// gd.eppo.int/taxon/PHTOOP (Consultado: 30 de agosto de 2018).

FAOSTAT (2018). Food and Agriculture Organization statistical database. Disponible en: http:// www.fao.org/statistics/es/ (Consultado: 30 agosto 2018).

Fenemore PG (1977). Oviposition of potato tuber moth, Phthorimaea operculella Zell. (Lep.: Gelechiidae); fecundity in relation to mated state, age, and pupal weight. New Zealand Journal of Zoology 4(2): 187-191. https://doi.org/10.1080/ 03014223.1977 .9517952

García-Mercet R (1925). La polilla de la patata. Boletín de la Real Sociedad Española de Historia Natural 25: 469-476.

Hajek A, Eilenberg J (2018). Natural enemies: an introduction to biological control. $2^{\mathrm{a}}$ Edición. Cambridge University, Cambrige, UK, 439 pp.

Harwalkar MR, Rananavare HD, Rahaikar GW (1987). Development of Trichogramma brasiliensis (Hym: Trichogrammatidae) on eggs of radiation sterilized females of potato tuberworm, Phthorimaea operculella (Lep.: Gelechiidae). Entomophaga 32(2): 159-162. https://doi.org/ 10.1007/BF02373126

Hou YY, Yang X, Zang LS, Zhang C, Monticelli LS, Desneux N (2018). Effect of oriental armyworm Mythimna separata egg age on the parasitism 
and host suitability for five Trichogramma species. Journal of Pest Science 91: 1181-1189. https://doi.org/10.1007/s10340-018-0980-2

Huang J, Hua HQ, Zhang F, Li YX (2018). Suitability assessment of three Trichogramma species in the control of Mythimna separata (Lep.: Noctuidae). Journal of Applied Entomology 142(12): 131-140. https://doi.org/10.1111/jen.12414

Kroschel J, Schaub B (2013). Biology and ecology of potato tuber moths as major pests of potato. En Insect Pests of Potato. Global Perspective on Biology and Management (Eds. Giordanengo P, Vincent C, Alyokhin A), pp. 165-192. Ed Elsevier, Massachusetts, USA.

Manly BFJ, Miller P, Cook LM (1972). Analysis of a selective predation experiment. The American Naturalist 106(952): 719-736. https://www.jstor. org/stable/2459502

MAPAMA (2017). Áreas demarcadas de Tecia solanivora en España. Disponible en: https:// www.mapa.gob.es/es/agricultura/temas/sanidad-vegetal/zonasdemarcadasteciaespana14junio2017_tcm30-381566.pdf (Consultado: 4 de diciembre de 2018).

Mills N (2010). Egg parasitoids in biological control and integrated pest management. En Egg parasitoids in agroecosystems with emphasis on Trichogramma (Eds Consoli FL, Parra JRP, Zucchi RA), pp 389-411. Ed Springer, Dordrecht, NL. https://doi.org/10.1007/978-1-4020-9110-0_15

Nurindah, Bronwen WC, Gordh G (1999a). Experience acquisition by Trichogramma australicum (Hym.: Trichogrammatidae). Australian Journal of Entomology 38: 115-119. https:// doi.org/10.1046/j.1440-6055.1999.00084.x

Pak GA (1988). Selection of Trichogramma for inundative biological control. Tesis Doctoral, Waganingen Agricultural University, Netherlands.

Pak GA (1990). Inundative release of Trichogramma for the control of cruciferous lepidoptera: preintroductory selection of an effective parasitoid. En: Diamondback moth and other crucifers pests: Proceedings of the second International Workshop (Ed Talekar NS). 10-14 December, Tainan, Taiwan. pp 297-308.
Pak GA, Kaskens JW, de Jong EJ (1990). Behavioural variation among strains of Trichogramma spp.: host species selection. Entomologia Experimentalis et Applicata 56(1): 91-102. https:// doi.org/10.1111/j.1570-7458.1990.tb01384.x

Perera-González S, Cubas-Hernández F, PadillaCubas Á, Carnero-Hernández A, HernándezBorges J, Asensio Ramos M. (2009). Ensayo de eficacia en el control de la polilla guatemalteca de la patata (Tecia solanivora) mediante la aplicación en campo de nematodos entomopatógenos. Cabildo Insular de Tenerife, 24 pp.

Pizzol J, Desneux N, Wajnberg E, Thiéry D (2012). Parasitoid and host egg ages have independent impact on various biological traits in a Trichogramma species. Journal of Pest Science 85(4): 489-496. https://doi.org/10.1007/s10340012-0434-1

Polaszek A (2010). Species diversity and host associations of Trichogramma in Eurasia. En Egg parasitoids in agroecosystems with emphasis on Trichogramma. (Eds. Consoli FL, Parra JRP, Zucchi RA), pp 237-266. Ed. Springer, Dordrecht, NL.

Pratissoli D, Parra JRP (2000). Desenvolvimento e exigências térmicas de Trichogramma pretiosum Riley, criados em duas traças do tomateiro. Pesquisa Agropecuária Brasileira 35(7): 12811288. http://dx.doi.org/10.1590/S0100-204X2 000000700001

Ricard I (2008). Statistical methods for insect choice experiments. Tesis Doctoral, Ecole Polytechnique Fédérale de Lausanne, CH. https:// doi.org/10.5075/epfl-thesis-4020

Rondon SI (2010). The potato tuberworm: a literature review of its biology, ecology, and control. American Journal of Potato Research 87(2): 149-166. https://doi.org/10.1007/s12230-0099123-x

Saour G (2004). Efficacy assessment of some Trichogramma species (Hym.: Trichogrammatidae) in controlling the potato tuber moth Phthorimaea operculella Zell. (Lep.: Gelechiidae). Journal of Pest Science 77(4): 229-234. https://doi.org/ 10.1007/s10340-004-0058-1

Trujillo-García E, Perera-González S (2014). Guía de lucha contra las plagas y enfermedades de la papa. Cabildo Insular de Tenerife, España. 24 pp. 
Trujillo-García E, Perera-González S (2017). Polilla guatemalteca de la papa (Tecia solanivora): identificación y control. Servicio Técnico de Agricultura y Desarrollo Rural, Cabildo de Tenerife, España. 8 pp.

Urquijo L. (1944). Ensayo del parasito útil Trichogramma minutum en la lucha contra la polilla de la patata. Boletín de Patología Vegetal y Entomología Agrícola 13: 281-300.

Van Lenteren JC (1980). Evaluation of control capabilities of natural enemies: Does art have to become science? Netherlands Journal of Zoology 30: 369-381. https://doi.org/10.1163/0028 29679X00467

Vásquez LA, Shelton AM, Hoffmann MP, Roush RT (1997). Laboratory evaluation of commercial Trichogrammatidae products for potential use against Plutella xylostella (L.) (Lep.: Plutellidae). Biological Control 9(2): 143-148. https:// doi.org/10.1006/bcon.1997.0528

Wade MR, Zalucki MP, Franzmann BA (2005). Influence of observer presence on pacific damsel bug behavior: who is watching whom?. Journal of Insect Behavior 18(5): 651-667. https:// doi.org/10.1007/s10905-005-7017-4
Wajnberg E (1993). Genetic variation in sex allocation in a parasitic wasp: variation in sex pattern within sequence of oviposition. Entomologia Experimentalis et Applicata 69: 221-229. https://doi.org/10.1111/j.1570-7458.1993. tb01745.x

Wajnberg E (2010) Genetics of the behavioral ecology of egg parasitoids. En: Egg parasitoids in agroecosystems with emphasis on Trichogramma (Eds. Consoli FL, Parra JRP, Zucchi RA), pp 149-165. Ed Springer Science+Business Media BV, Dordrecht, NL.

Zimmerman O, Wührer B (2010). The South American Tomato Moth, Tuta absoluta, a new pest in Germany: An assessment of biological control options. En: Report on the $28^{\text {th }}$ Annual Meeting of the Working Group "Beneficial Arthropods and Entomopathogenic Nematodes" (Eds. Herz A, Ehlers RU). Journal of Plant Diseases and Protection 117: 88. https://doi.org/ 10.1007/BF03356340

(Aceptado para publicación el 27 de mayo de 2019) 